\title{
The Impact of Different Induction Immunosuppressive Therapy on Long-Term Kidney Transplant Function When Measured by lothalamate Clearance
}

\author{
Tambi Jarmi ${ }^{\mathrm{a}, \mathrm{c}}$, Samir Khouzam ${ }^{\mathrm{a}}$, Nitika Shekhar ${ }^{\mathrm{a}}$, Meray Hosnia ${ }^{\mathrm{a}}$, Launia White ${ }^{\mathrm{b}}$, \\ David O. Hodge ${ }^{\text {b }}$ Martin L. Mai ${ }^{\mathrm{a}}$, Hani M. Wadei ${ }^{\mathrm{a}}$
}

\begin{abstract}
Background: Improvement in short-term outcomes after kidney transplant has been achieved by using different induction and maintenance therapeutic approaches. Long-term outcomes have not matched the expectations of the transplant stakeholders. Our study aimed to address the early impact of induction agents on long-term outcome of kidney transplant when measured by iothalamate clearance.
\end{abstract}

Methods: All adult kidney transplant recipients between January of 2012 and December of 2016 were reviewed. Six hundred fortynine patients were divided into three groups based on the induction agent (basiliximab, alemtuzumab, and thymoglobulin). Protocoled 4 months and 48 months kidney allograft function evaluations with iothalamate clearance test were compared among the three groups.

Results: Patients who received basiliximab were significantly older with no difference among African American and Caucasians. The 48 months assessment showed significant decline in median iothalamate clearance in basiliximab group at $49 \mathrm{~mL} / \mathrm{min}$ vs. alemtuzumab group $64.8 \mathrm{~mL} / \mathrm{min}$ and thymoglobulin $60 \mathrm{~mL} / \mathrm{min}$ with $\mathrm{P}=0.007$. The basiliximab group developed a significant higher proteinuria measured by spot urine to creatinine ratio at 48 months.

Conclusions: The use of basiliximab as an induction agent for kidney transplant is associated with significant decline in kidney function 4 years post transplantation when measured by iothalamate clearance.

Keywords: Induction; Iothalamate; Allografts

Manuscript submitted October 21, 2020, accepted November 11, 2020

Published online December 18, 2020

aDepartment of Transplant, Mayo Clinic Florida, Jacksonville, FL 32224, USA bDepartment of Health Sciences Research, Mayo Clinic Florida, Jacksonville, FL 32224, USA

${ }^{c}$ Corresponding Author: Tambi Jarmi, Department of Transplant, Mayo Clinic Florida, 4500 San Pablo Road, Jacksonville, FL 32224, USA.

Email: Jarmi.Tambi@mayo.edu

doi: https://doi.org/10.14740/jocmr4369

\section{Introduction}

With development of immunosuppressive medications and major advances in clinical care, short-term outcomes after kidney transplant are noticeably better $[1,2]$. However, the main challenge is how to improve long-term outcomes $[3,4]$. The introduction of potent and selective agents for the initiation of immunosuppression, as induction therapy, has reduced the incidence of acute rejection early post transplantation and improved 1-year graft survival [5-11]. However, long-term graft survival was less linked to induction therapy and more to maintenance immunosuppressive regimen and existence of medical comorbidities. The Kidney Disease Improving Global Outcomes (KDIGO) guidelines recommend basiliximab as a first-line induction therapy across all types of donor-recipient profiles to reduce acute rejection risk and allograft loss [12, 13]. However, there has been a lack of randomized clinical trials comparing induction agents when recipients are receiving a maintenance immunosuppressive therapy with tacrolimus (TAC) and mycophenolic acid (MPA) with or without steroids; and the recommendations are mainly made on the basis of meta-analysis data $[11,14]$. Since the incidence of acute rejection is less than $10 \%$ in most reported for cause biopsies, the sample size required for randomized clinical trials that could determine small differences in observed outcomes between basiliximab, alemtuzumab and thymoglobulin has been estimated to be between 1,600 and 7,000 [15]. Such studies would be difficult to conduct from logistics standpoints. Given such an obstacle, we reviewed 649 kidney transplant recipients at Mayo Clinic Florida from 2012 to 2016 and compared the long-term kidney transplant function, based on the induction therapy used at the time of transplantation. Kidney allograft function was measured by iothalamate as a standard clearance test [16].

\section{Materials and Methods}

\section{Patient population}

After obtaining required Institutional Review Board (IRB) approval, all adult patients who received kidney transplant 
at Mayo Clinic Florida between January 2012 and December 2016 were reviewed in this study. Seventy hundred ninetyseven adult patients were identified. We excluded patients who received combined kidney and other solid organ transplant, kidney after previous solid organ transplant, and second or third kidney transplant. Totally, 649 patients were included in this study.

\section{Study design}

The study is a retrospective analysis of kidney transplant recipients at a single transplant center, Mayo Clinic Florida Transplant Center. The study was conducted in compliance with the ethical standards of the responsible institution on human subjects as well as with the Helsinki Declaration, and received IRB approval before initiation. Patients were divided into three groups based on the induction agent used at the time of transplantation and according to immunosuppressive therapy protocols approved and adopted by Mayo Clinic Transplant Enterprise. Group 1 represent low immunological risk and received basiliximab (patients 65 years of age and older and/or two haplotype-matched donors or zero mismatch); group 2 received alemtuzumab (patients 64 years of age and younger and/or no detected donor specific antibodies (DSA) or detected DSA but with mean florescence intensity (MFI) < 2,000); group 3 represent high immunological risk and received thymoglobulin (patients 64 years of age and younger with DSA at MFI $\geq 2,000$ or positive acceptable flow cytometry cross match with DSA at any value). Groups 1 and 3 will receive maintenance steroid with $5 \mathrm{mg}$ prednisone on daily basis. Group 2 will not receive maintenance steroid. All transplant recipients were maintained on TAC and MPA for long-term immunosuppression.

\section{Data points}

Patients' demographic data were collected. Allografts functional data points on 4 and 48 months were collected and included: Iothalamate clearance which is a standard of care at our center and performed on all kidney transplant recipients within the first 4 months and at 48 months post-transplant. Urine protein to creatinine ratio, time to first rejection, TAC level with first rejection and pathology score of first rejection.

\section{Statistical analysis}

Categorical variables were presented as the number of subjects and the percentages. Medians were used to summarize the continuous variables. Overall comparisons among the groups for categorical variables were completed using the $\chi^{2}$ test. The Kruskal-Wallis test was used to compare continuous variables. The cumulative probability of a rejection was estimated using the Kaplan-Meier method. These curves were compared between groups using the log-rank test. All analyses were completed using SAS version 9.4 (Cary, NC).

\section{Results}

\section{Demographics and comorbidities}

Out of the 649 reviewed patients, group 1 has 149 patients received basiliximab, group 2 has 264 received alemtuzumab and group 3 has 236 received thymoglobulin. Patients induced with basiliximab were significantly older as expected. African Americans patients were represented in each group with no significant differences when compared to White patients (Table 1). We analyzed the pre-transplant diabetes history (type I and II) and found no significant differences among the three induction groups (Table 1). All reviewed patients were also analyzed based on their smoking history documented on the initial evaluation visit pre-transplant and no differences in the incidence of smoking among the three groups (Table 1). Body mass index (BMI) was reviewed, all accepted patients for transplantation is recommended to have BMI of 32 and less according to Mayo Clinic Transplant evaluation protocol and all reviewed patients showed no differences in their BMI calculated at the time of transplantation (Table 1).

\section{Kidney transplant long-term function based on induction agents}

The protocoled 4 months iothalamate clearance showed no difference among the three groups (alemtuzumab $65.7 \pm 26 \mathrm{~mL} /$ $\mathrm{min}$, basiliximab $62.8 \pm 29.6 \mathrm{~mL} / \mathrm{min}$ and thymoglobulin 68.6 $\pm 29.6 \mathrm{~mL} / \mathrm{min}, \mathrm{P}=0.13$ ). However, it was significantly lower, at 48 months, in patients induced with basiliximab $52.6 \pm 30$ in comparison to alemtuzumab $64.8 \pm 34$ and thymoglobulin $61 \pm 30.1(\mathrm{P}<0.007)$ (Fig. 1a). When we compared the three groups at 48 months after excluding all patients who received deceased kidney allografts from donors with kidney donor profile index (KDPI) greater than $85 \%$, we found similar significant decline in allograft function at 48 months in patients induced with basiliximab $(52 \mathrm{~mL} / \mathrm{min})$ when compared with alemtuzumab $(64.0 \mathrm{~mL} / \mathrm{min})$ and thymoglobulin $(61 \mathrm{~mL} / \mathrm{min})$ (Fig. 1b). Overall, 48 months proteinuria, measured by spot urine protein to creatinine ratio, among the three groups was not high. However, the median protein creatinine ratio was significantly higher in patients induced with basiliximab (0.2) when compared to patients induced with alemtuzumab (0.1) and thymoglobulin $(0.1)(\mathrm{P}<0.03)$ (Fig. 2).

\section{Rejection, cytomegalovirus (CMV) and polyomavirus (BK) incidence}

Although, Banff classification of first diagnosed rejection based on protocoled and indicated biopsies was not different among the three groups, patients induced with basiliximab showed $7.4 \%$ increase incidence of IIB rejection when compared to alemtuzumab $2.9 \%$ and thymoglobulin $0.0 \%$ with $\mathrm{P}$ $<0.3$ (Table 2). Time for first rejection (Fig. 3a) was not different among the three induction groups $(\mathrm{P}>0.05)$ and tac- 
Table 1. Patients Demographics

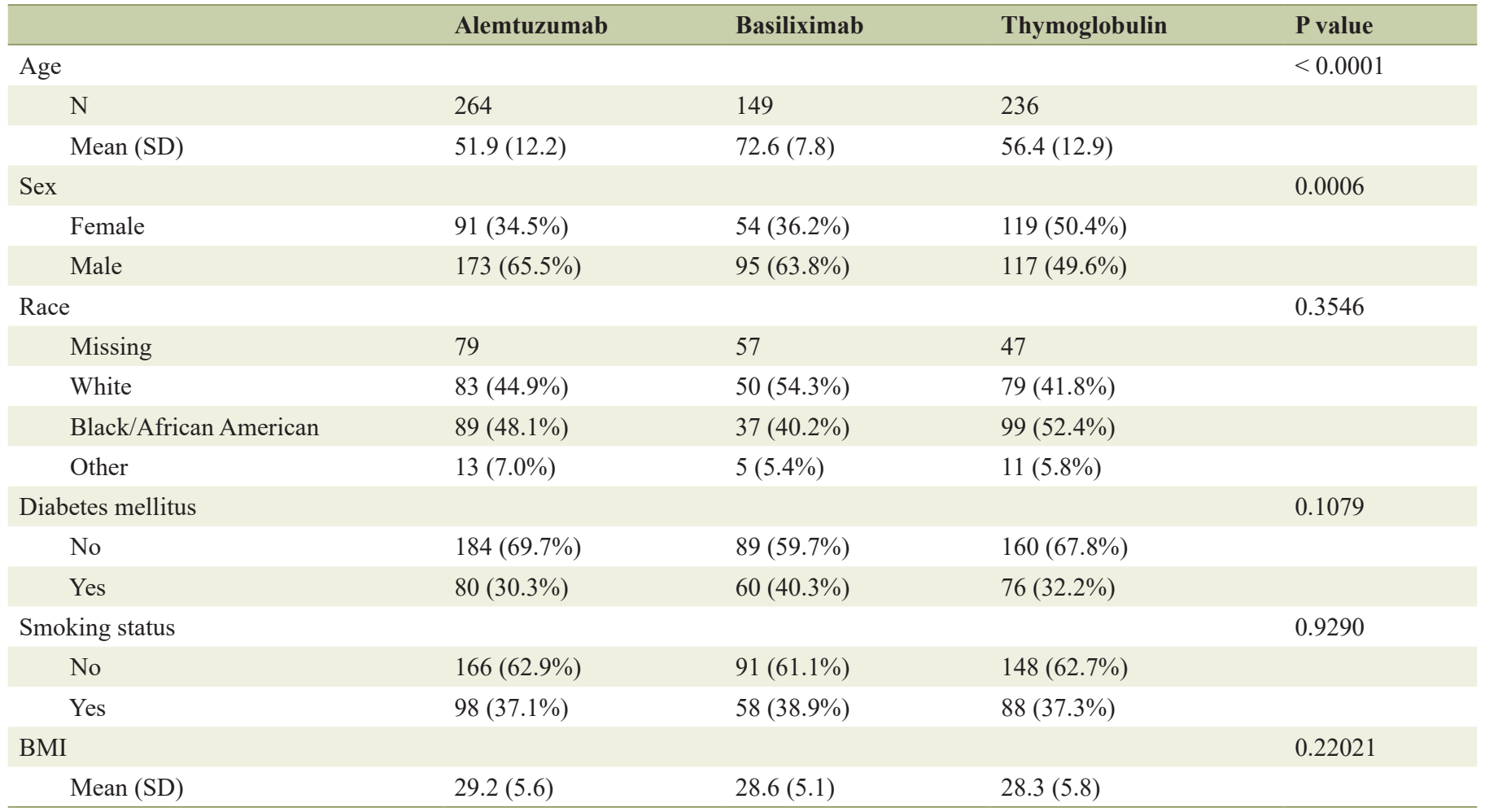

Totally, 649 patients were enrolled. Age was significantly higher in group received basiliximab as expected based on immunosuppressive induction protocol (patients 65 years of age and older). Thymoglobulin induction group has equal female and male distribution when compared to alemtuzumab and basiliximab.

rolimus level (mean \pm standard deviation $(\mathrm{SD}))$ at the time of first rejection was at $7.7 \pm 3.8 \mathrm{ng} / \mathrm{mL}$ with thymoglobulin, 6.8 $\pm 2.5 \mathrm{ng} / \mathrm{mL}$ with alemtuzumab and $7.3 \pm 3.1 \mathrm{ng} / \mathrm{mL}$ with basiliximab group (Fig. 3b) $(\mathrm{P}>0.05)$. Among the three groups of patients, incidence of viremia was higher with thymoglobulin and viremia incidence was higher with alemtuzumab. The overall incidence of CMV and BK was not significant among the three groups (Fig. 4).

\section{Discussion}

Conventionally, the monoclonal antibodies directed against the activated interleukin-2 (IL-2) receptor such as basiliximab, have been usually reserved for low to moderate immunologic risk patients [17-19], whereas the depleting agents, such as thymoglobulin and alemtuzumab, have been used in high-risk recipients receiving kidney transplantation [7, 20, 21]. Our study is a retrospective analysis of kidney allograft's long-term function based on immunosuppressive induction agents used at time of transplant. We found a significant drop in iothalamate clearance when measured at 48 months post-transplant. However, the 4 months iothalamate clearance was similar among all patients. This change from equivalent iothalamate clearance at 4 months between the groups to a drop in iothalamate clearance at 48 months in basiliximab group may be less related to donor factors and more to immunosuppressive approach. In or- der to correct for the donor factors, we repeated the 48 months analysis after excluding all recipients from all three groups who received kidney allografts from deceased donors with KDPI > $85 \%$. The secondary analysis still showed significant drop in iothalamate clearance in patients received basiliximab for induction when compared to alemtuzumab and thymoglobulin.

The Cochrane Collaboration published a meta-analysis of randomized controlled trials that compared IL-2 receptor antagonist, such as basiliximab, induction with placebo and thymoglobulin [22]. Acute rejection rates were lower with IL-2 receptor antagonist versus placebo and graft loss was reduced. However, thymoglobulin was not more effective in preventing rejection, and the safety profile favored IL-2 receptor antagonist induction. Based largely on these findings, KDIGO guidelines recommended that induction therapy with a biological agent be a routine part of the initial immunosuppressive regimen and that an IL-2 receptor antagonist agent to be the first-line therapy [23]. However, little information is available about the long-term effects of the IL-2 receptor antagonist basiliximab; most of the published studies addressed the short-term benefits and risks of basiliximab in terms of reducing incidence of rejection and monitoring for side effects [17]. In our study, we reviewed the long-term, 5 years, kidney allografts outcome when using basiliximab vs. alemtuzumab and thymoglobulin for induction. We showed no difference in time to first diagnosed rejection, no difference in the severity of first diagnosed rejection based on Banff classification 

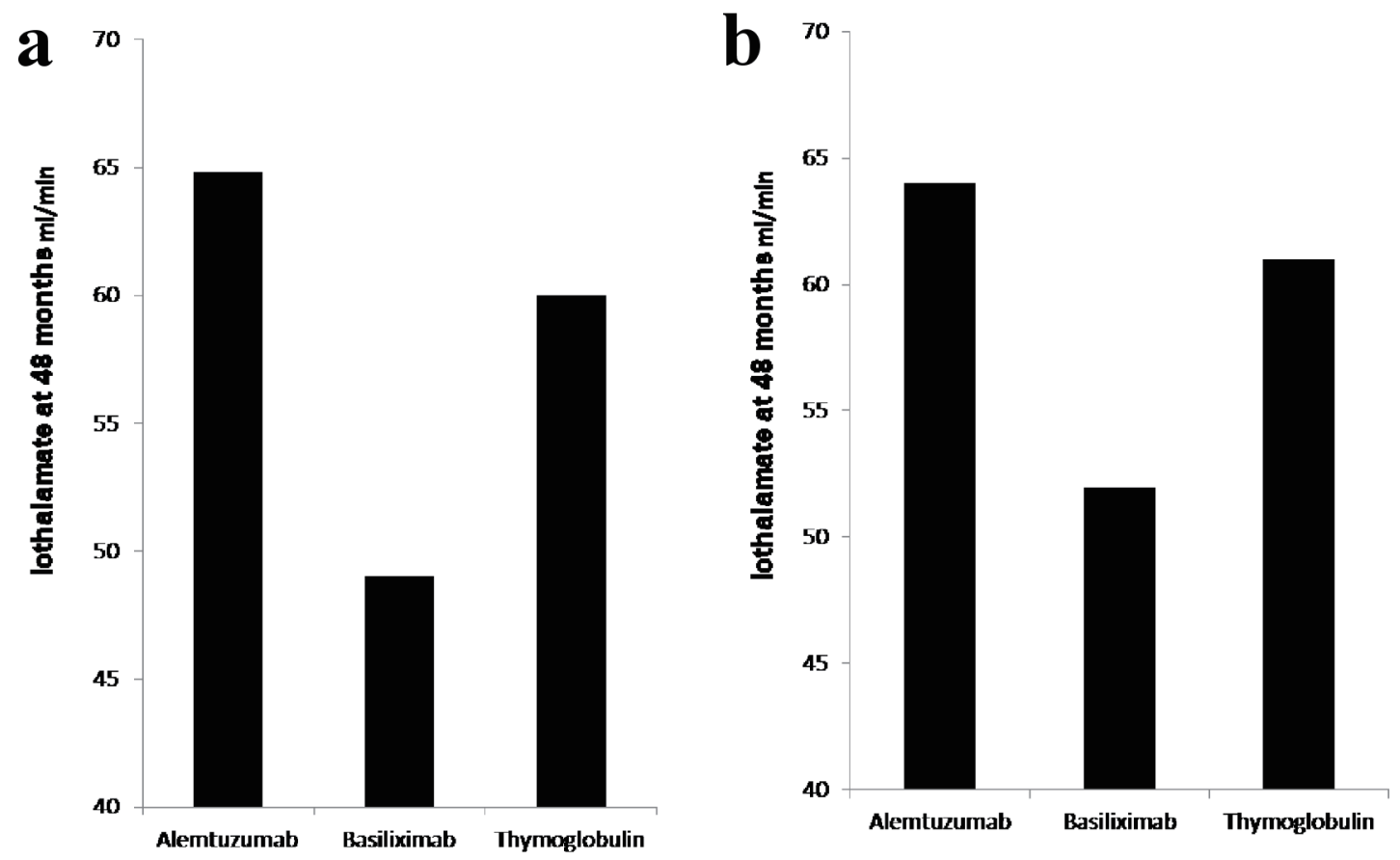

Figure 1. (a) lothalamate clearance at 48 months. Recipients induced with basiliximab (149 patients) showed significant decline in iothalamate clearance at 49 when compared to alemtuzumab (264 patients) at 64.8 and thymoglobulin (236 patients) at 60 with $\mathrm{P}=0.007$. (b) lothalamate clearance at 48 months, after excluding of recipients from donors with KDPI $>85 \%$. Recipients induced with basiliximab (129 patients) showed significant decline in iothalamate clearance at 52 when compared to alemtuzumab (256 patients) at 64 and thymoglobulin (229 patients) at 61 with $\mathrm{P}=0.02$.

[24] among patients who were induced with basiliximab when compared to alemtuzumab and thymoglobulin. We accounted for possible donor factors represented by high KDPI by repeating the analysis after excluding all patients who received

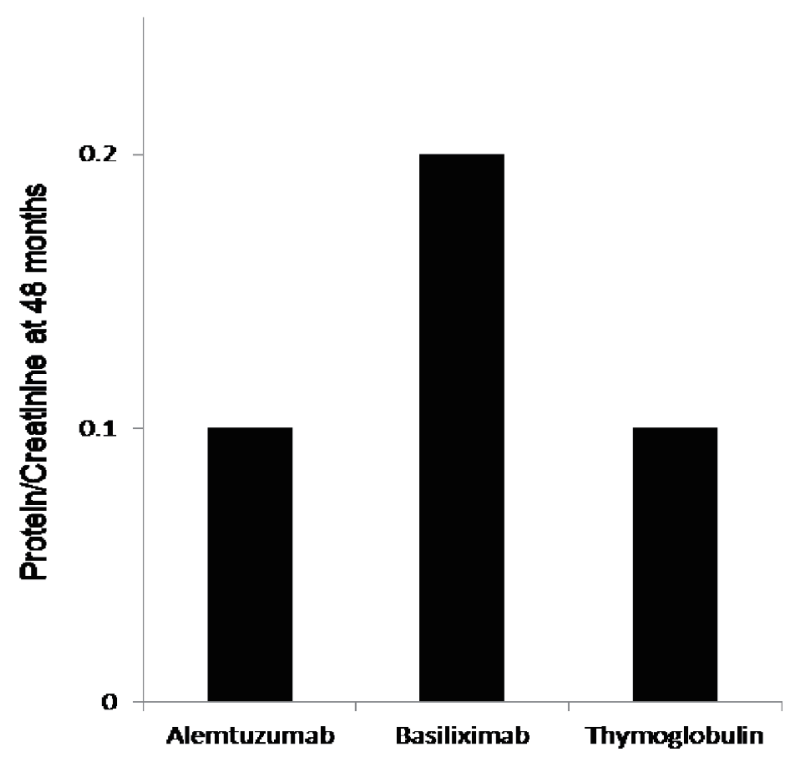

Figure 2. The 48 months protein creatinine ratio measurement showed significantly elevated mean protein/creatinine when measured at 48 months at 0.2 when compared to protein/creatinine of 0.1 with both alemtuzumab and thymoglobulin when used for induction, $\mathrm{P}<0.03$. kidney allografts from donors with KDPI $>85 \%$. The iothalamate clearance [25] at 5 years post-transplant was significantly lower in patients who received basiliximab for induction when compared to alemtuzumab and thymoglobulin. This results remains the same even after we excluded old recipient of kidney allograft from donors with KDPI $>85 \%$. Despite the similarities in serology and pathology findings among the three groups, the significant difference in iothalamate clearance at 5 years post-transplant in the basiliximab group is alarming. We could argue that the patients who received basiliximab had chronic smoldering and subclinical cellular injury that resulted in such decline in kidney allograft function and the increase in proteinuria [26, 27].

In this study, we analyzed the incidence of viral infection, mainly CMV and BK, among the three groups of patients based on the induction agent. Multiple studies have shown an increased risk of viral infection when T-cell depleting agent is used for induction therapy versus basiliximab [28-30]. However, we found no significant difference in the incidence of either CMV or BK disease among the different induction groups.

\section{Conclusions}

This study demonstrated potential effect of the induction agent used at the time of kidney transplantation on 5 years allograft function when measured by iothalamate clearance. Using basiliximab resulted in lower iothalamate clearance and increased proteinuria 5 years post-transplant. 
Table 2. Rejection Severity

\begin{tabular}{llll}
\hline & Alemtuzumab $(\mathbf{n}=\mathbf{3 5})$ & Basiliximab $(\mathbf{n}=\mathbf{2 7})$ & Thymoglobulin $(\mathbf{n}=\mathbf{2 7})$ \\
\hline $\begin{array}{l}\text { Rejection severity } \\
\text { Borderline }\end{array}$ & $19(55.9 \%)$ & $14(51.9 \%)$ & \\
IA & $8(23.5 \%)$ & $7(25.9 \%)$ & $21(77.8 \%)$ \\
IB & $5(14.7 \%)$ & $2(7.4 \%)$ & $5(18.5 \%)$ \\
IIA & $1(2.9 \%)$ & $1(3.7 \%)$ & 0.3813 \\
IIB & $1(2.9 \%)$ & $2(7.4 \%)$ & $1(3.7 \%)$ \\
\hline
\end{tabular}

First diagnosed rejection classified according to Banff 2017 criteria showed no significant difference among the three induction agents. Patients who received basiliximab for induction had $7.4 \%$ increase incidence of IIB rejection when compared to $2.9 \%$ with alemtuzumab and $0 \%$ with thymoglobulin $(P=0.3)$.

\section{Acknowledgments}

Thanks to the Mayo Clinic Transplant Center.

\section{Financial Disclosure}

No associated finances that need to be disclosed. The study required no funding.

\section{Conflict of Interest}

None to declare.

\section{Informed Consent}

The study is a retrospective analysis and did not require informed consent.

\section{Author Contributions}

Tambi Jarmi contributed to the hypothesis, methods and design, data collection and analysis, data and results interpretation, and manuscript writing. Samir Khouzam: data collection. Nitika Shekhar: data collection. Meray Hosni: data collection. Launia White: data analysis. David O. Hodge: data analysis. Martin L. Mai: data and results interpretation, and manuscript

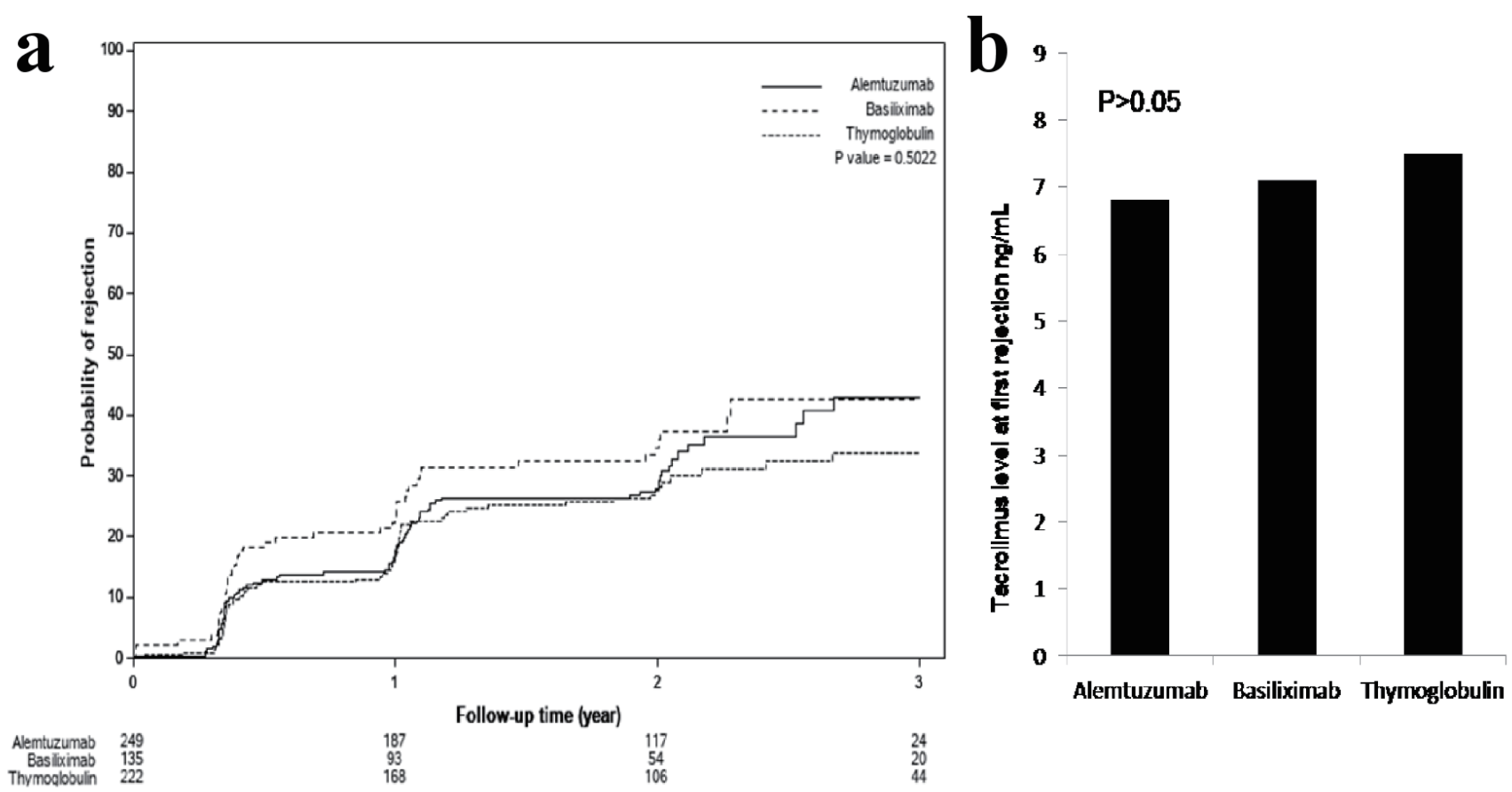

Figure 3. (a) Time to first rejection, based on protocoled or indicated biopsies, showed no significant differences among the three groups $(P>0.05)$. (b) Tacrolimus level checked within the $24-48 \mathrm{~h}$ prior to first diagnosed rejection showed acceptable therapeutic levels and no differences among the three groups: $7.7 \pm 3.8 \mathrm{ng} / \mathrm{mL}$ with thymoglobulin, $6.8 \pm 2.5 \mathrm{ng} / \mathrm{mL}$ with alemtuzumab and $7.3 \pm 3.1 \mathrm{ng} / \mathrm{mL}$ with basiliximab group $(P>0.05)$. 


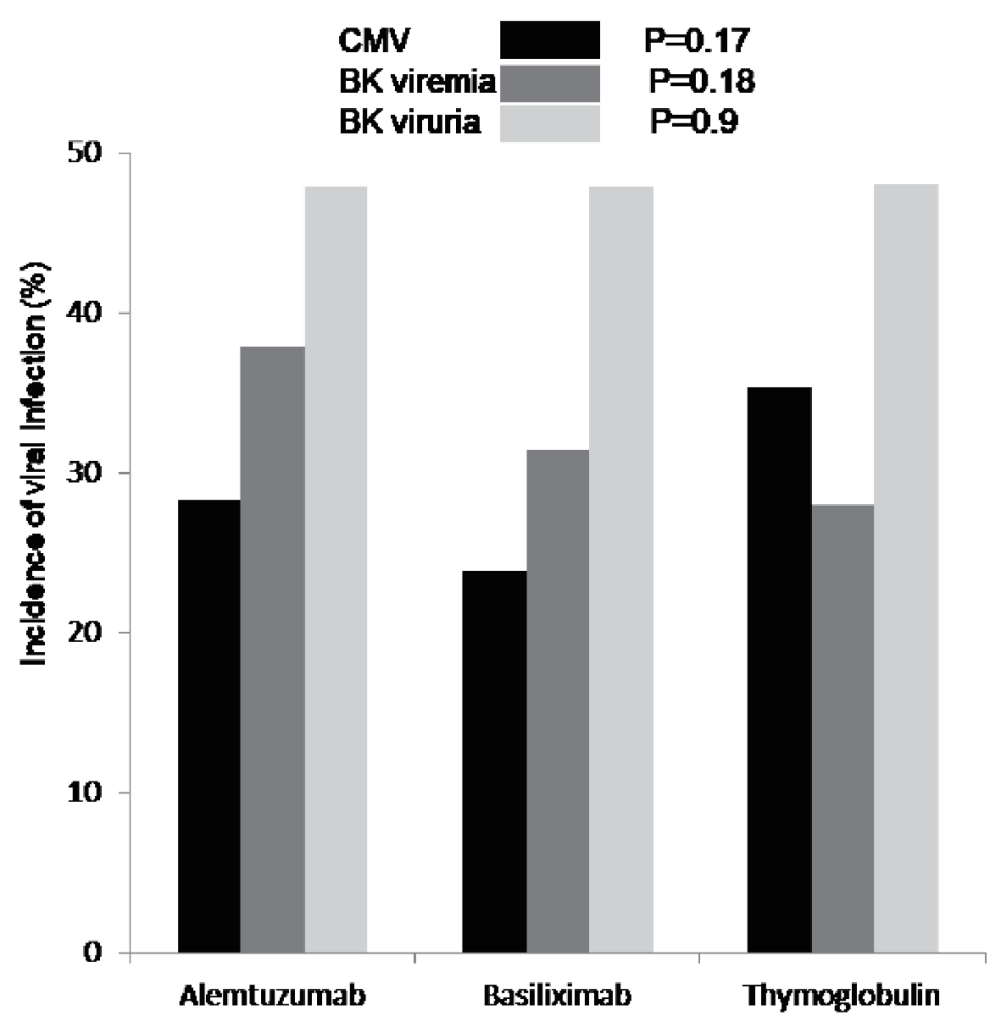

Figure 4. First diagnosed CMV viremia (black), based on screening test and/or symptoms, was reported as percentage of patients who tested positive and showed no difference among the three groups $(P=0.17)$. Incidence of $B K$ viremia (grey) was higher in patients who received alemtuzumab for induction but not significant $(P=0.18)$. Incidence of $B K$ viruria (white) was similar in all patients group $(P=0.9)$. CMV: cytomegalovirus; BK: polyomavirus.

writing. Hani M. Wadei: data and results interpretation, and manuscript writing.

\section{Data Availability}

Any inquiries regarding supporting data availability of this study should be directed to the corresponding author.

\section{References}

1. Gaston RS. Improving long-term outcomes in kidney transplantation: towards a new paradigm of post-transplant care in the United States. Trans Am Clin Climatol Assoc. 2016;127:350-361.

2. Wang JH, Skeans MA, Israni AK. Current status of kidney transplant outcomes: dying to survive. Adv Chronic Kidney Dis. 2016;23(5):281-286.

3. Patel AK, Samaniego M. The struggle for optimization of long-term outcomes after kidney transplantation. Adv Chronic Kidney Dis. 2016;23(5):280.

4. Brennan DC. Long-term trends in allograft survival. Adv Chronic Kidney Dis. 2006;13(1):11-17.

5. Halloran PF. Immunosuppressive drugs for kidney transplantation. N Engl J Med. 2004;351(26):2715-2729.
6. Hellemans R, Bosmans JL, Abramowicz D. Induction therapy for kidney transplant recipients: do we still need Anti-IL2 receptor monoclonal antibodies? Am J Transplant. 2017;17(1):22-27.

7. Hill P, Cross NB, Barnett AN, Palmer SC, Webster AC. Polyclonal and monoclonal antibodies for induction therapy in kidney transplant recipients. Cochrane Database Syst Rev. 2017;1:CD004759.

8. Khalkhali HR, Ghafari A, Hajizadeh E, Kazemnejad A. Risk factors of long-term graft loss in renal transplant recipients with chronic allograft dysfunction. Exp Clin Transplant. 2010;8(4):277-282.

9. Tanriover B, Jaikaransingh V, MacConmara MP, Parekh JR, Levea SL, Ariyamuthu VK, Zhang S, et al. Acute rejection rates and graft outcomes according to induction regimen among recipients of kidneys from deceased donors treated with tacrolimus and mycophenolate. Clin J Am Soc Nephrol. 2016;11(9):1650-1661.

10. Van Loon E, Senev A, Lerut E, Coemans M, Callemeyn J, Van Keer JM, Daniels L, et al. Assessing the complex causes of kidney allograft loss. Transplantation. 2020;104(12):2557-2566.

11. Wiseman AC. Induction therapy in renal transplantation: why? What agent? What dose? We may never know. Clin J Am Soc Nephrol. 2015;10(6):923-925.

12. Isakova T, Nickolas TL, Denburg M, Yarlagadda S, Wein- 
er DE, Gutierrez OM, Bansal V, et al. KDOQI US Commentary on the 2017 KDIGO clinical practice guideline update for the diagnosis, evaluation, prevention, and treatment of Chronic Kidney Disease-Mineral and Bone Disorder (CKD-MBD). Am J Kidney Dis. 2017;70(6):737751.

13. Mandelbrot DA, Reese PP, Garg N, Thomas CP, Rodrigue JR, Schinstock C, Doshi M, et al. KDOQI US Commentary on the 2017 KDIGO clinical practice guideline on the evaluation and care of living kidney donors. Am J Kidney Dis. 2020;75(3):299-316.

14. Williams AM, Barrett M, Smith AR, Kathawate RG, Woodside KJ, Sung RS. Variable benefits of antibody induction by kidney allograft type. J Surg Res. 2020;248:6981.

15. Willoughby LM, Schnitzler MA, Brennan DC, Pinsky BW, Dzebisashvili N, Buchanan PM, Neri L, et al. Early outcomes of thymoglobulin and basiliximab induction in kidney transplantation: application of statistical approaches to reduce bias in observational comparisons. Transplantation. 2009;87(10):1520-1529.

16. Dowling TC, Frye RF, Fraley DS, Matzke GR. Comparison of iothalamate clearance methods for measuring GFR. Pharmacotherapy. 1999;19(8):943-950.

17. Ponticelli C. Basiliximab: efficacy and safety evaluation in kidney transplantation. Expert Opin Drug Saf. 2014;13(3):373-381.

18. Wang $\mathrm{K}, \mathrm{Xu} \mathrm{X}$, Fan M. Induction therapy of basiliximab versus antithymocyte globulin in renal allograft: a systematic review and meta-analysis. Clin Exp Nephrol. 2018;22(3):684-693.

19. Yakubu I, Ravichandran B, Sparkes T, Barth RN, Haririan A, Masters B. Comparison of alemtuzumab versus Basiliximab induction therapy in elderly kidney transplant recipients: a single-center experience. J Pharm Pract. 2019:897190019850934.

20. Gundroo A, Zachariah M, Singh N, Sharma R. Alemtuzumab (Campath-1H) experience in kidney transplantation what we have learned; current practices; and scope for the future? Curr Opin Organ Transplant. 2015;20(6):638-642.

21. Morgan RD, O'Callaghan JM, Knight SR, Morris PJ.
Alemtuzumab induction therapy in kidney transplantation: a systematic review and meta-analysis. Transplantation. 2012;93(12):1179-1188.

22. Webster AC, Ruster LP, McGee R, Matheson SL, Higgins GY, Willis NS, Chapman JR, et al. Interleukin 2 receptor antagonists for kidney transplant recipients. Cochrane Database Syst Rev. 2010;1:CD003897.

23. Kidney Disease: Improving Global Outcomes Transplant Work Group. KDIGO clinical practice guideline for the care of kidney transplant recipients. Am J Transplant. 2009;9(Suppl 3):S1-155.

24. Roufosse C, Simmonds N, Clahsen-van Groningen M, Haas M, Henriksen KJ, Horsfield C, Loupy A, et al. A 2018 Reference Guide to the Banff Classification of Renal Allograft Pathology. Transplantation. 2018;102(11):17951814.

25. Soveri I, Berg UB, Bjork J, Elinder CG, Grubb A, Mejare I, Sterner G, et al. Measuring GFR: a systematic review. Am J Kidney Dis. 2014;64(3):411-424.

26. Baldwin WM, 3rd, Valujskikh A, Fairchild RL. Mechanisms of antibody-mediated acute and chronic rejection of kidney allografts. Curr Opin Organ Transplant. 2016;21(1):7-14.

27. Sellares J, de Freitas DG, Mengel M, Reeve J, Einecke $\mathrm{G}$, Sis B, Hidalgo LG, et al. Understanding the causes of kidney transplant failure: the dominant role of antibodymediated rejection and nonadherence. Am J Transplant. 2012;12(2):388-399.

28. Luan FL, Samaniego M, Kommareddi M, Park JM, Ojo AO. Choice of induction regimens on the risk of cytomegalovirus infection in donor-positive and recipientnegative kidney transplant recipients. Transpl Infect Dis. 2010;12(6):473-479.

29. Pai D, Mann DM, Malik A, Hoover DR, Fyfe B, Mann RA. Risk factors for the development of BK virus nephropathy in renal transplant recipients. Transplant Proc. 2015;47(8):2465-2469.

30. Bayraktar A, Catma Y, Akyildiz A, Demir E, Bakkaloglu $\mathrm{H}$, Ucar AR, Dirim AB, et al. Infectious complications of induction therapies in kidney transplantation. Ann Transplant. 2019;24:412-417. 